\title{
Over half all patients develop short-term root sensitivity after periodontal therapy
}

\author{
What is the prevalence of root sensitivity after periodontal therapy?
}

von Troil B, Needleman I, Sanz M. A systematic review of the prevalence of root sensitivity following periodontal therapy. J Clin Periodontol 2002; 29(Suppl. 3):S173-S177

Data sources MEDLINE and EMbase reference lists from relevant articles were hand-searched and a hand-search was made of selected journals dated up to April 2001.

Study selection No randomised controlled trials (RCT) nor quasirandomised clinical trials were identified, so the review focused on cross-sectional and prospective clinical trials in which periodontitis patients were periodontally treated and the intensity of root sensitivity was evaluated.

Data extraction and synthesis Data extraction was carried out in duplicate using a predetermined appraisal form.

Results The prevalence of root sensitivity was 9-23\% before and 54$55 \%$ after periodontal therapy. An increase in the intensity of root sensitivity occurred 1-3 weeks following therapy, after which it decreased.

Conclusions There were insufficient RCT to adequately address the stipulated question. Based on the scarce evidence from only two studies, root sensitivity occurs in approximately half of patients following subgingival scaling and root planing. The intensity of root sensitivity increases for a few weeks after therapy, after which it decreases. In clinical practice, it may be recommended that, prior to treatment, patients should be made aware of the potential for root sensitivity. In research, there is a need for RCT and prospective studies with both short and long follow-up periods. Studies are also required that investigate the effects and the relationship of root instrumentation with the aetiology of root sensitivity; the efficacy of preventive and therapeutic regimes for root sensitivity; the incidence and severity of root sensitivity by subjective patient-reporting; and the response to different modes of stimuli. Furthermore, protocols should follow the criteria used in dentine hypersensitivity studies.

Address for correspondence: Birgitta von Troil, Albertinkatu 24A, FIN-00120 Helsinki, Finland. E-mail: birgitta.vontroil@kolumbus.fi

\section{Commentary}

Chronic periodontal disease is an infectious disease characterised by inflammation and subsequent destruction of the supporting structures of the teeth. Management is generally by a combination of surgical and non-surgical treatment (ie, scaling and root planing). The success of periodontal therapy is usually determined by clinical outcomes such as maintenance or improvement in clinical attachment levels. When making clinical decisions, however, patients should be informed about all the potential risks and benefits, and the probability of those risks and benefits. This includes not only a decreased risk of future tooth loss, but negative sequelae of therapy such as gingival recession and root sensitivity.

Root sensitivity is distinct from dentinal hypersensitivity in that the former is a result of periodontal therapy or periodontitis, the latter of hydrodynamic stimulation. Root sensitivity can significantly affect an individual's quality of life, in that dietary choices become limited, effective oral hygiene may be impeded and, as a result of recession, aesthetics can be negatively impacted.

In their systematic review, von Troil and colleagues examined studies reporting the prevalence of root sensitivity. Of the two studies that met the authors' inclusion criteria, neither was randomised. Only one had a control group. Each measured root sensitivity differently. In one study, the outcome was determined by clinical means (mechanical force or air stimulation), in the other as patient reports in the form of a visual analogue scale.

Regardless, the prevalence was similar: 55 and $54 \%$ of patients reported an increase in root sensitivity 1 week after surgical and/or non-surgical periodontal therapy. Although the intensity of sensitivity remained statistically significant up to 4 weeks posttherapy, it gradually decreased.

Despite the fact that both the disease and its treatment are known primary aetiological factors in root sensitivity, this systematic search of the literature revealed only two studies. This is yet another example of an area of research focusing solely on clinical outcomes, ignoring patient-based measures. These findings underline the need for well-designed clinical trials of both long and shortterm duration. These trials must use both standardised clinical and patient-based outcomes. In providing informed consent to our patients, we need to know the likelihood of developing postoperative root sensitivity, and the likely duration. Data from patient-based outcomes are also essential, since they determine the potential economic burden this condition actually represents.

\section{Practice point}

- Current best evidence suggests that over half of all patients suffer with root sensitivity after periodontal treatment.

\author{
Debora Matthews \\ Head, Division of Periodontics, Dalhousie University, Halifax, \\ Nova Scotia, Canada
}

Evidence-Based Dentistry (2003) 4, 63.

doi:10.1038/sj.ebd.6400199 